\title{
The Phasehood Analysis of Chinese Nominal Phrases and NP Ellipsis
}

\author{
Cunying Fan \\ Department of English Language \& Literature, Gyeongsang National University, Gyeongnam, South Korea; \\ Foreign Languages Teaching \& Research Department, Qufu Normal University, Shandong, China
}

\begin{abstract}
Bošković, in his paper Now I'm a phase, now I'm not a phase: On the variability of phases with extraction and ellipsis, puts forward two hypotheses about phasehood and ellipsis from a contextual perspective: a. Only phases and complements of phase heads can undergo ellipsis. b. The highest projection in a TNP (Traditional Noun Phrase) is a phase. This paper tentatively tests whether these two hypotheses can be used in the analysis of structures and ellipsis of Chinese NPs. After analysis, the paper discovers that Bošković's hypotheses can explain the structure and ellipsis of Chinese simple NPs but they cannot account for Chinese complex NPs well. Based on this, the paper modifiers Bošković's hypotheses slightly: a. Only phases and complements of phase heads can undergo ellipsis. b. Each functional projection over a NP in Chinese is a phase.
\end{abstract}

Index Terms—phasehood, ellipsis, Chinese simple NPs, Chinese complex NPs, NP structure

\section{INTRODUCTION}

Different from Chomsky's rigid approach to phasehood, Bošković (2014) argues for a particular contextual approach to phasehood where the highest projection in the extended projection of a major (i.e. lexical) category functions as a phase. His argument involves two domains: extraction and ellipsis. Based on these two domains, Bošković puts forward two hypotheses: a. Only phases and complements of phase heads can undergo ellipsis. b. The highest projection in a TNP (Traditional Noun Phrase) is a phase. These two hypotheses can be expressed as two approaches respectively: highest-phrase-as-a-phase approach and ellipsis-constrained-by-phase approach.

In this paper, the syntactic structure of Chinese NPs and ellipsis within Chinese NPs are approached from two broad categories: simple NPs and complex NPs. The purpose of doing this is to tentatively test whether Bošković's two hypotheses about phasehood and ellipsis can be used in the analysis of the structure of Chinese NPs with respect to ellipsis within NPs. Bošković's hypotheses are studied from two broad categories of Chinese NPs: Chinese simple NPs and Chinese complex NPs. Section 2 discusses the analysis of Chinese simple NPs. Chinese complex NPs are investigated in section 3. Finally in section 4, a brief conclusion about the application of Bošković's hypotheses in Chinese Nps is given.

\section{Chinese Simple NPS AND THEIR ELLIPSIS}

Bošković $(2008,2012)$ argues based on a number of syntactic and semantic crosslinguistic generalizations that languages without articles, like Chinese, lack DP. Chierchia (1998) makes the same claim for languages like Chinese based on very different considerations regarding the semantics of TNPs. In this paper, I will adopt Bošković and Chierchia's NP approach to article-less languages and explore the syntax structure and ellipsis of Chinese NPs in terms of Bošković's phasehood theory and ellipsis theory.

Considering the modifiers in front of nouns, Chinese simple NPs can be divided into four basic kinds: Numeral + Classifier $+\mathrm{NP}$, Demonstrative + Classifier $+\mathrm{NP}$, Possessor $+D e+\mathrm{NP}$ and Adjective $+D e+\mathrm{NP}$. In this paper, these different kinds of NPs are discussed from the perspective of their syntactical structure and their ellipsis.

\section{A. Chinese Simple NPs: Numeral + Classifier $+N P$}

In Chinese, nouns can be modified by numerals to express singular or plural quantity. Different from numerals in English NPs, numerals in Chinese NPs have to be followed by classifiers which are determined by the semantic property of the nouns after them. 'In Chinese whenever there is a numeral, there has to be an overt classifier' (Y. R. Chao, 1968). For example:

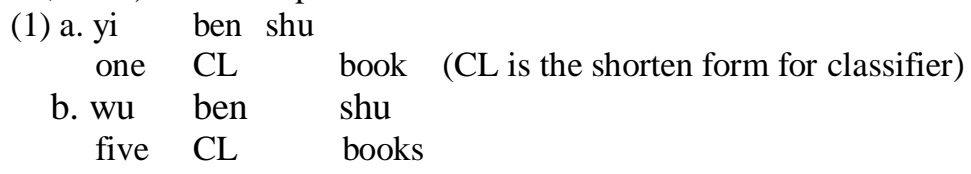

How can we analyze these Chinese numeral NPs? Do they share the same structure as English numeral NPs about which Ionin and Matushansky (2004a, b) argue that numerals act as nominal modifiers, taking NP complements? I assume that the structure of Chinese numerals NPs must be different from that of English numeral NPs since Chinese 
numeral NPs have overt classifiers within them. So if we want to analyze the structure of Chinese NPs with numerals, the first thing we have to consider is the status of the overt classifiers.

About the function and syntactical position of classifiers in Chinese numeral and demonstrative NPs, many linguists have discussed and most tend to regard them as a functional head of a classifier projection.

Cheng and Sybesma (1999) suggest that the classifier in Chinese takes up the functions of D in article languages, including:

(a) The classifier has an individualising and singularising function. It links the description of the NP to a particular entity $=>$ the deictic function.

(b) Classifiers are like Ds in the sense that they type-shift predicates into arguments =>the'subordinator' function (Szabolcsi 1994).

(c) The classifier head realises the iota operator (which is the equivalent of the definite article) (Partee 1987) $=>$ encoding of definiteness.

Based on this, Cheng and Sybesma (1999) claim that Chinese CLP (Classifier Phrase/Projection) may be equivalent to the DP and thus dominant the NP. They think that the classifier is an appropriate candidate to be a functional head to project a structure. Many other linguists also hold this opinion, such as, Tang (1990) argues that CLP is a superstructure of a Chinese NP. After settling down the structural role taken by the classifier, we will come to the syntactical position taken by the numeral prior to the classifier. In their analysis about Turkish NPs, Bošković and Sener notice that ellipsis inside bare objects with numerals is disallowed:

(2) *Pelin her gün [beş elma] ye-r, Can-sa [iki elma] ye-r. P.-nom every day five apple eat-pres J.-nom-however two eat-aor Pelin eats five apples every day, while John eats two apples.

However in Turkish, NP/N' ellipsis is possible if Num (shorten form for Numeral) is accompanied by CL, in contrast to (2), where there is no CL and ellipsis is disallowed.

(3) Pelin her gün [üç tane elma] ye-r, Can-sa [iki tane elma] ye-r.

P.-nom every day three CL apple eat-aor J.-nom-however two CL eat-aor.

Pelin eats three apples every day, whereas John eats two apples.

Bošković and Sener assume that Turkish numerals that are accompanied by a CL project larger structure than those without a CL provides us. Consider (4)

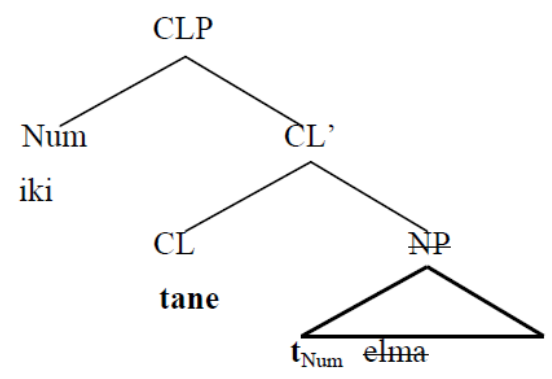

Bošković and Sener suggest that numerals are base-generated and Num moves to SpecCLP in the presence of overt classifiers.

Following Bošković and Sener's analysis of Turkish numerals accompanied by overt classifiers, we can assume that Chinese numeral NPs which require overt classifiers have the same structure with structure (4). That is: Num occupies the position of SpecCLP in Chinese numeral NPs. However because Turkish numerals can be followed by both covert classifiers and overt classifiers, Bošković and Sener suggest that numerals are base-generated and Num moves to SpecCLP in the presence of overt classifiers. But in Chinese, numerals have to be followed by overt classifiers. So no movement of numerals is required and numerals merge directly into SpecCLP.

Actually in his discussion of the necessity of an overt classifier in the Chinese numeral NPs, Au-Yeung (2001) mentions that there is a need to fill in the empty CL with a classifier item in Chinese. It is structurally ungrammatical when the SpecCLP is filled with a numeral and the head CL is left empty. This argument not only supposes that the classifier can project a phrase but also supposes that the numeral occupies the position of SpecCLP. The structure of example $(1 \mathrm{~b})$ can be analyzed in the following way: 
(5)

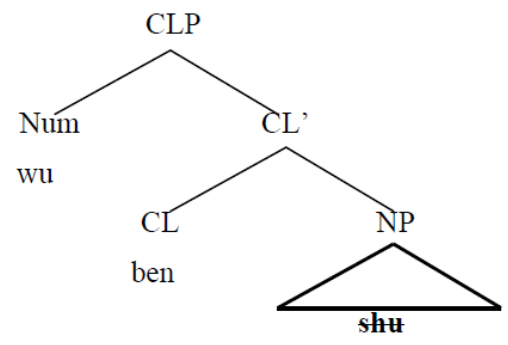

In the introduction part, I have mentioned that a number of authors have argued that Chinese lacks DP and TNPs are typically NPs in Chinese. About the phasehood of NPs, (Bošković, 2014) argues that 'It turns out that the phasehood status of NP depends on whether or not another phrase dominates it...'. Considering the structure of Chinese numeral NPs, such as (5), NPs are dominated by another structure projected by overt classifiers. In terms of Bošković's highest-phrase-as-a-phase approach, NPs are not phases instead CLPs are phases because NPs are dominated by phrase CLPs.

Similar to Turkish numeral NPs with overt classifiers, Chinese numeral NPs in which numerals must be followed by overt classifiers can also allow ellipsis (Saito, Lin \& Murasugi, 2008). After the ellipsis, the numeral and the classifier are stranded.

Look at an example:

(6) Suiran Zhangsan mai-le san ben shu, dan Lisi ma-le [wu ben shu]. though Zhangsan buy-Perf three CL book, but Lisi buy-Perf five CL book

Zhangsan bought three books, but Lisi bought five. (Masao Ochi, 2012)

The structure of the numeral NP in the example (6) is the same as the structure (5), we can show here again with the ellipsis showing in structure (7).

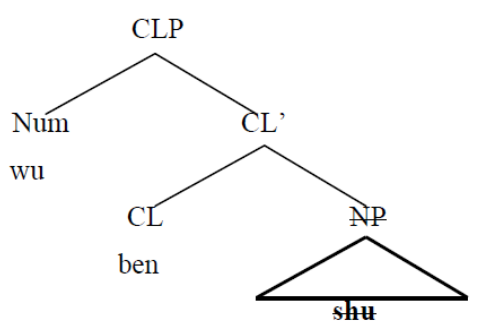

Bošković (2014) hypothesizes that 'regarding ellipsis, it is argued that ellipsis is phase-constrained: only phases and complements of phasal heads can in principle undergo ellipsis.' And recall that CLP is a phase in structure (7). So only two phrases can possibly undergo ellipsis in this structure: CLP which is a phase and NP which is the complement of the phase CLP. From the perspective of Bošković's ellipsis-constrained-by-phase approach, the ellipsis of NP 'shu' involves the ellipsis of the complement of the head CL 'ben'.

In Chinese numeral NPs, there is another interesting phenomenon needs our attention: Though numerals must be accompanied by overt classifiers, classifiers can be proceeded by a covert or null numeral. When the numeral in front of the classifier is null, it can express a singular concept. Under this circumstance, both the NP and the CLP can undergo ellipsis. Consider example (8) and structure (9).

(8) a. Zhangsan mai-le ben shu, Lisi ye mai-le [ben shel].

b. Zhangsan mai-le ben shu, Lisi ye mai-le [ben shu]

Zhangsan buy-Perf CL book, Lisi too buy-Perf CL book

Zhangsan bought one book, Lisi bought one book too.

(9)

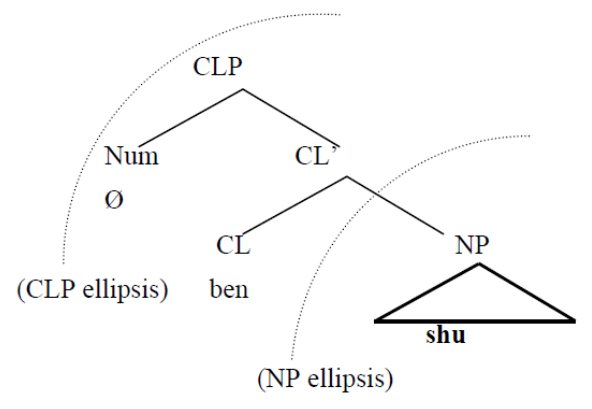


Similarly, CLP functions as a phase in structure (9). Sentence (8a) is the ellipsis of the NP 'shu' is the ellipsis of the complement of the phasal head. Sentence (8b) is the ellipsis of the phase itself.

A short summary of this section: In Chinese numeral NPs, the overt classifier functions as the head of the CLP and chooses NP as its complement. The numeral is in the position of SpecCLP. According to Bošković's hypotheses about phasehood, CLP is a phase in Chinese numeral NPs. And Bošković argues that ellipsis in phase-governed: only phase itself and complement of phase head can undergo ellipsis. So in Chinese numeral NPs, both NP and CLP have the possibility of being elided. In this sense, we can say that Bošković's hypotheses about phasehood and ellipsis can account for the analysis of Chinese NPs: Numeral + Classifier + NP.

\section{B. Chinese Simple NPs: Demonstrative + Classifier + NP}

There is another kind of Chinese NPs which include overt classifiers. In this kind of NPs, demonstratives appear in front of classifiers. Here the demonstrative only limitedly refers to demonstrative pronouns ' $z h e$ ' (this) ' $n a$ '(that). Since overt classifiers appear both in Chinese numeral NPs (i.e. numeral + classifier + NP) and Chinese demonstrative NPs (i.e. demonstrative + classifier + NP), next we will try to analyze whether these two structures share similar structure.

In literature, opinions about the analysis of the structure of 'Demonstrative + Classifier + NP' vary dramatically. Given a simple example:

(10) a. Wo xihua zhe ben shu, ta xihua [na ben shu.]

b. Wo xihua zhe ben shu, ta xihua [na ben shu.]

I like this CL book, he like that CL book.

I like this book, but he likes that book.

Gao (1994) assumes the demonstratives and the CLP together constitute the DemP and the DemP functions as the specifier of the head noun. (10a) can be analyzed as (11) b:

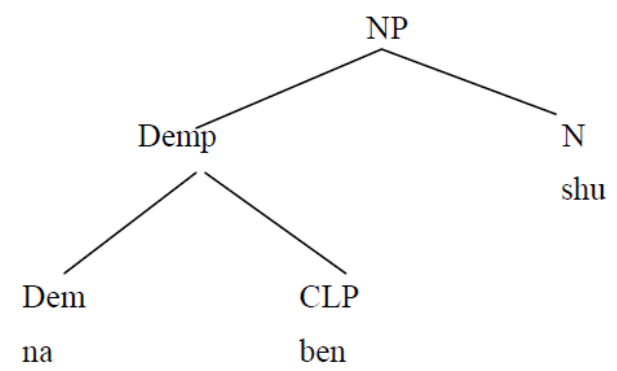

In Bošković's highest-phrase-as-a-phase approach, in structure (11) NP is the phase and N is its head. And in terms of Bošković's ellipsis theory, $\mathrm{N}$ 'shu' which functions as a phase head in this structure cannot be elided. However actually the noun 'shu' can be deleted and the grammaticality of the ellipsis of the noun 'shu' in (10b) helps us to discard Gao's analysis.

Xue and McFetridge (1998) assume that Dem is the head of DemP and selects NP as its complement. And this NP consists of CLP and a noun.

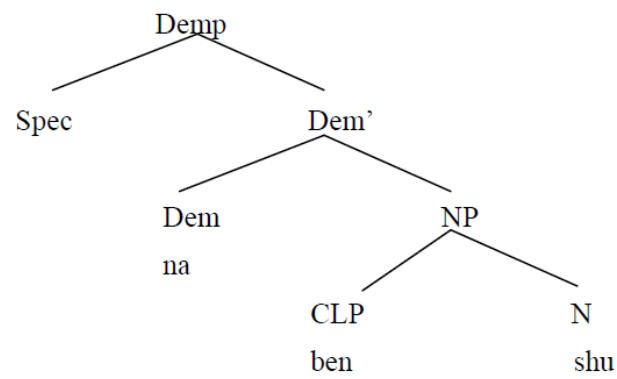

Whether this analysis is reasonable can be accounted from two aspects.

Firstly, we can illustrate it with Bošković's ellipisis hypothesis, which 'privileges only two domains for ellipsis: the phase itself and the complement of a phase head. There is, e.g., no natural way of privileging the complement of a complement of a phase head.' In Xue and McFetridge's analysis, Demp is the highest phrase and so it is the phase. In this phase, Dem ' $n a$ ' is the head and the NP is the complement of this head. The N 'shu' is the complement of the complement of the phase head. So in this analysis the $\mathrm{N}$ 'shu' cannot undergo ellipsis. This is contrary to the grammaticality of sentence (10b).

Secondly, Fukui (1995) argues that only functional categories project up to a certain XP level. This XP level closes the structure in such a manner that nothing that is interpretatively within the XP level can show up outside the 
c-commanding domain of $\mathrm{X}$, unless $\mathrm{X}$ has Case to assign to its specifier position. Under this assumption, he finds that Demonstratives in English are like functional heads in the sense that they close the nominal domain properly. No element can appear to the left of the demonstrative:

(13) a. this book

$$
\text { b. * John's this book }
$$

(14) a. that lecture

b. *yesterday's that lecture

While in Chinese, more than one modifier can appear to the left of the demonstrative. Consider the following Chinese examples:

(15) Wo de na ben shu

I $\mathrm{K}$ that $\mathrm{CL}$ book ( $\mathrm{K}$ is used to refer to that ' $d e$ ' is a functional particle)

that book of mine

(16) Wo de hongse de na ben shu

$\mathrm{I} \mathrm{K}$ red $\mathrm{K}$ that $\mathrm{CL}$ book

that red book of mine

Since the Chinese demonstrative does not close off the projection, this lends support to the claim that the demonstrative in Chinese cannot be a functional head which can project a structure.

From these two aspects, we can say that Xue and McFetridge's analysis is also not appropriate. Since both Gao's and Xue and McFetridge's analysis cannot ensure the production of the correct sentence, we have to choose an alternative analysis. We can follow Bošković and Sener's analysis of Turkish numeral NPs with overt classifiers, and analyze the structure of 'Demonstrative + Classifier + N' as a CLP with a CL head and the demonstrative occupy the position of SpecCLP.

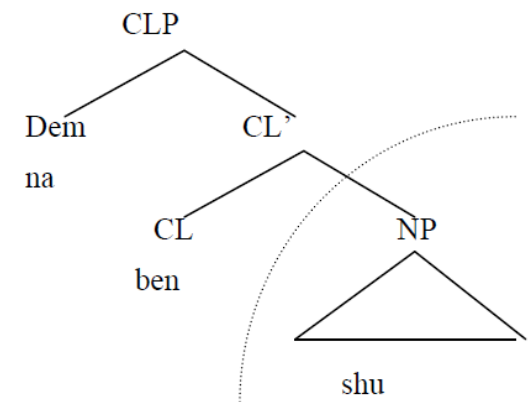

In this context, CLP is the phase (CLP is the highest phrase in the TNP), hence NP, its complement, can be elided.

From the above analysis, we find that Chinese NP structures with a classifier (Numeral + Classifier + N/Demonstrative + Classifier+ N) have a similar feature: There is a higher structure CLP (which is a phase) over the NP. This higher structure has a functional head - a classifier and this head chooses NP as its complement. Other modifying elements such as numerals or demonstratives occupy the position of SpecCLP. Thus we get the following unified structure about Chinese NPs with classifiers:

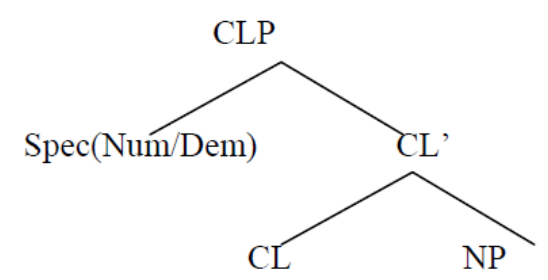

In addition, CLP is the highest phase in this unified structure and the NP ellipsis involves the ellipsis of a phsal complement or a phase itself. Under this situation, Bošković' two hypotheses about phases and ellipsis have the force of explanation for Chinese numeral and demonstrative NPs.

\section{Chinese NPs: Possessor $+D e+N P$}

When considering Chinese NPs with possessors, Partee (2005) notes that while the English sentence (19) has the presupposition 'Zhangsan has exactly three sweaters,' the Chinese sentence (20) doesn't have that exhaustive presupposition:

(19) Zhangsan's three sweaters (Zhangsan has exactly three sweaters)

(20) Zhangsan de [san jian maoyi] 
Zhangsan $\mathrm{K}$ three $\mathrm{CL}$ sweater

Zhangsan's three sweaters (Zhangsan has exactly three sweaters or Zhangsan has more than three sweaters)

Bošković (2012) shows that the exhaustive presupposition is not present in NP languages in this context. This directly shows that Chinese language is a NP language.

Another special feature about Chinese possessive NPs is that there is a marker element which is an attributive particle $d e$ following the possessor (No matter the possessor is a noun or a pronoun) in Chinese possessive NPs. Particles are functional parts of speech that can't stand alone by themselves. Chinese makes liberal use of these particles, as it lacks inflection and verbal expressive tones like the Western non-tonal languages. The particle we met up with most frequently thus far is de.

If we are to analyze the structure of Chinese possessive NPs, we should decide the status of possessors and particle ' $d e$ ' respectively.

First let's consider the status of possessors. As noted by Jackendoff (1971), NP ellipsis is possible in English possessive NPs. After the ellipsis, the possessor will be stranded.

(21) I have read Bill's book, but I haven't read [John's bøek].

In his analysis, Jackenoff (1971) assumes that the possessor occupies the position of SpecDP. According to Bošković's phase theory and ellipsis theory, DP is the highest phrase and so it is the phase in this structure and the ellipsis of the NP is the ellipsis of the complement of DP.

Similarly when NP ellipsis occurs in Chinese possessive NPs, the possessor also strands. The stranding possessor shows that perhaps the possessor in Chinese possessive NPs share the same position with the English ones. Here I adopt this assumption, but how do we analyze the position of the stranding article de which is stranded together with the possessor after NP ellipsis?

When analyzing NP ellipsis in Japanese, Bošković oberserves that the topic article survives ellipsis and he argues that 'since the particle survives ellipsis it appears that we need to place it in a separate projection outside of the ellipsis site, which is the NP.' Takahashi (2011) holds the same argument that the topic particles in Japanese are located in the head position of KP which takes NP as its complement. The possessor is then adjoined to KP: [KP Possessor [K' [K[NP]]]]

Since the topic article can serve as the head of the KP in Japanese, it follows that the attributive particle after the possessor in Chinese NPs can similarly function as a head.

Here is an example:

(22) Zhe shi wo de shu, na shi [ta de shu].

This is my $\mathrm{K}$ book, that is his $\mathrm{K}$ book.

This is my book and that is his book.

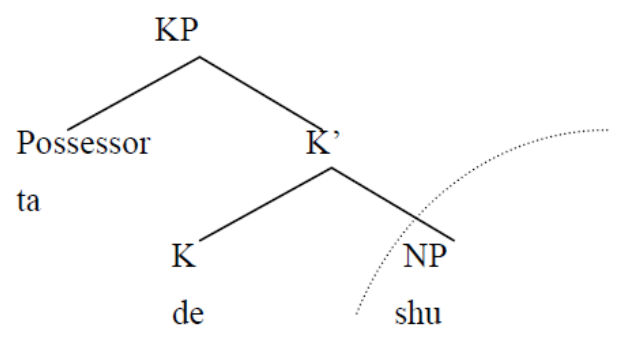

In structure (23), KP is the highest projection in the TNP hence it is a phase. According to Bošković's ellipsis theory, (23) involves ellipsis of the complement of a phase head. The analysis in (23) predicates the sentence in (22) to be well-formed.

To sum up, in this section I assume that the article de heads a KP and takes a NP complement in Chinese possessive NPs which have an attributive article $d e$. Furthermore, the possessor occupies the position of SpecKP. NP ellipsis in this structure is about the deletion of the complement of the phase head $\mathrm{K}$ de, with both the possessor and the article de stranded. From this aspect, we can say that Bošković phase theory and ellipsis theory can explain the structure of Chinese possessive NPs.

\section{Chinese Simple NPs: Adjective + De + NP}

Adjectives can be used to modify the noun. However opinions about the structural position of adjectives are different. According to Abney (1987), prenominal adjectives should be taken as heads of noun phrases. However Pysz (2006) argues 'for adjectives in DPs, they may be adjuncts to the maximal projection of the nominal head, to its intermediate projection or to the nominal head itself.' Scholars like Crisma (1993), Cinque (1995), and Laenzlinger (2000) deem that attributive adjectives should be specifiers of noun phrases. In this paper I accept the assumption of adjective-as-a-specifier hypothesis.

Considering Chinese NPs with an attributive adjective, its structure is similar to that of English adjective NPs. But one difference exists: a particle de inserts between the adjective and the noun. We recall that in the analysis of Possessor $+D e+\mathrm{NP}$ structure, de is taken as the functional head of KP and possessor occupies SpecKP. In the analysis of 
Chinese adjective NP, I integrate the opinion of particle ' $d e$ ' projecting a functional KP with the opinion of adjective-as-a-specifier hypothesis mentioned by many scholars mentioned above. Having this assumption, we can analyze the following example in this way:

(24) Dajia xihua shangliang de ren, bu xihua [lengku de ren].

Everybody like kind $\mathrm{K}$ people, not like cruel K people.

Everybody likes kind people, and don't like cruel people.

(25)

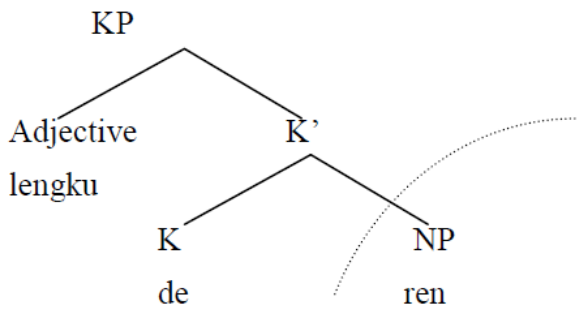

In terms of Bošković's ellipsis theory, NP in adjective Chinese NPs can be deleted because it is the complement of the phase head ( KP is the highest phrase and so it is a phase).

Though semantically different, Possessor $+D e+\mathrm{NP}$ and Adjective $+\mathrm{De}+\mathrm{NP}$ in Chinese share the same syntax structure: Particle de functions as a head of KP that is the highest phrase projected by the noun. The possessor and the adjective occupy the SpecKP position.

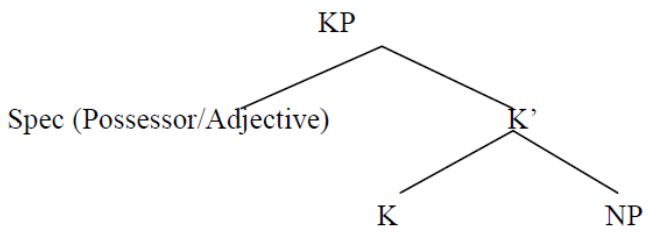

KP projected by the functional head $d e$ is the highest phase in this unified structure and the NP ellipsis involves the ellipsis of the complement of the functional head K. From this perspective, Bošković's two hypotheses about phases and ellipsis account for the analysis of Chinese possessive and adjective NPs.

\section{E. Conclusion}

Up to now, we have discussed four basic kinds of Chinese NPS: Numeral+Classifier+NP, Demonstrative + Classifier $+\mathrm{NP}$, Possessror $+D e+\mathrm{NP}$ and Adjective $+D e+\mathrm{NP}$. From the analysis of them, we find that there is a similarity among these structures: There is a higher projection projected by a functional head (the functional head can be either a classifier or an attributive article $d e$ ) over the NP. If we use a general term FuntionalP to stand for the higher phrases (CLP and KP) over NPs, we can get a unified structure:

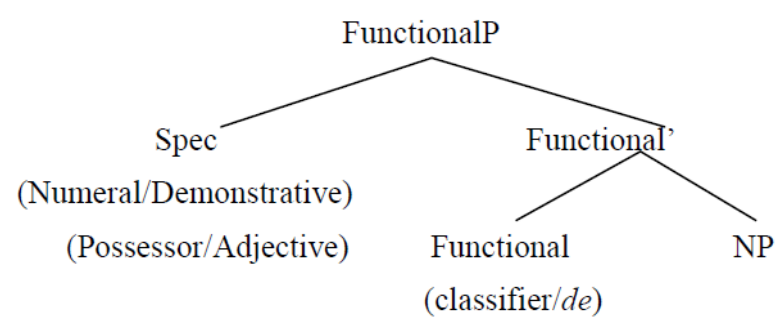

According to Bošković, FunctionalP is the highest phrase hence it is a phase. Ellipsis of FuntionalP itself and complement of the phase head Functional is possible.

\section{ChINESE COMPLEX NPS}

The discussion in section 2 is about simple Chinese NPs. In the syntactic structure of this kind of NPs, there is only one FunctionalP above NP. In terms of Bošković's phase theory, this FunctionalP is a phase. And in terms of his ellipsis theory, the NP functioning as the complement of the phase head can undergo ellipsis. From the above analysis, we discover that Bošković's two hypotheses about phase and ellipsis can be applied to the explanation of Chinese simple NPs. However in Chinese, many complex NPs with more than one modifier in front of the noun exist. Can Bošković's 
two hypotheses have the similar force of explanation in the analysis of Chinese complex NPs? This will be the focus of this section.

Bošković (2009a, 2012) observes that word order within TNP is generally freer in NP than DP languages. The reason for this is that the richer structural configuration of DP languages imposes syntactic restriction on word order within TNP in DP languages that are not found in NP languages due to the lack of the syntactic structure that is responsible for these restrictions. This is true in Chinese NPs. Tough the modifiers are normally put in front of the noun in Chinese, the word order of the modifiers is relatively free than that of some other DP language, say English.

Some examples can be easily found in Chinese. In principle, any order of numerals, demonstratives, possessives, and adjectives is allowed in Chinese. A few examples are listed:

(28) a. Possessor + De > Numeral +Classifier $>>N P$

Zhangsan de san ben shu

Zhangsan $\mathrm{K}$ threeCL book

Zhangsan's three books

b. Possessor+De $>>$ Adjective+De $>>N P$

Zhangsan de hongse de chenshan

Zhangsan $\mathrm{K}$ red $\mathrm{K}$ shirt

Zhangsan's red shirt

c. Demonstrative+classifier $>>$ Possessor+De $>>N P$

Zhe bu Lian de dianying

This CL Lian $\mathrm{K}$ movie

this movie of Lian's

d. Demonstrative+Classifier $>>$ Adjective + De $>>N P$

na bu hongsede paoche

that CL red sport-car

e. Possessors+ De >>demonstrative+ classifier $>>$ adjective+De $>>$ NP

Zhangsan de na bu hongse che

Zhangsan $\mathrm{K}$ that $\mathrm{CL}$ red car

Of course, more kinds of similar structures are permitted in Chinese NPs. However no matter what kind of structure is it, there is more than one functional projection over the nominal phrase. Then how can we analyze these kinds of complex NPs?

Take an example, where to allocate elements that appear to the left of the numeral and classifier (e.g. the demonstratives, possessors, etc.). At first blush, this does not seem to be a problem as one can have demonstratives and possessors adjoined to the Classifier Phrase. However, interestingly, the presence of modifiers to the left of the classifier may alter the referential properties of the noun phrase. Assuming that modifiers are adjoined to the Classifier Phrases cannot account for the change in referentiality. So this pushes us to think they are located in a higher projection superior to the projection of classifiers. Just like what we have analyzed, all the four basic structures of Chinese NPs sharing that same syntactic structure FunctionalP. So we can assume that in the complex NPs, every modifier belong to a functional phrase heading by a functional particle (the classifier and the attributive particle de). So in Chinese complex NPs, more than one functional phrase projected over the noun.

Next let's try to check whether Bošković's hypotheses work for the analysis of Chinese complex NPs with more than one functional phrase. Consider one example (in this example, some elements of the NPs in the later part of the sentence can be omitted):

(29) Zhangsan mai-le san ben Qiaomusiji de shu,

Zhangsan buy-Perf three CL Chomsky $\mathrm{K}$ book.

a. Lisi ye mai-le [ san ben Qiaomusiji de-shu].

b. Lisi ye mai-le [san ben Qiammusiji de shu.]

c. Lisi ye mai-le [san ben Qiammsiji de shu].

Lisi also buy-Perf [three CL Chomsky $\mathrm{K}$ book]

Zhangsan bought three Chomsky's books and Lisi also bought three Chomsky's books.

Having the assumption that in Chinese complex NPs, every modifier belongs to a functional phrase heading by a functional particle, The structure of the later part of (29) can be analyzed in the following way: 
(30)

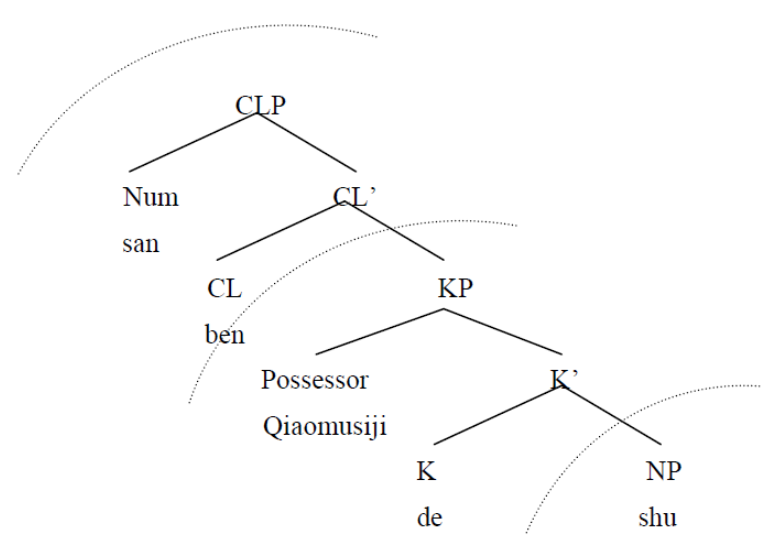

Recall that highest phrase is a phase, so in structure (30) CLP is the phase. And the constraints about ellipsis are that only the phase itself and complement of the phase head can undergo ellipsis. So we can explain the grammaticality of (29 c) which is the ellipsis of the phase CLP. We can also account for the grammaticality of (29b) which is the ellipsis of KP since KP is the complement of the phase CLP. But how can we explain the grammaticality of (29a). If we assume that CLP is the phase and then NP becomes the complement of the complement of the phase head. This is not allowed in Bošković's theory about ellipsis. Shall we say Bošković's theory doesn't work for the analysis of Chinese complex NPs. Not necessarily. Because Chinese NPs has a special feature, different from other language, that is, each projection over NP is headed by a functional head (a classifier or a particle De). Taking this into account, we need to modifier Bošković's hypothesis a little:

(31) a. Only phases and complements of phase heads can undergo ellipsis.

b. Each functional projection over NP in Chinese is a phase.

So according to this modified hypothesis, we can say that both CLP and KP are phases in (30). We can use the modified hypothesis in (30) to explain the grammaticality of (29a), (29b) and (29c). (29c) is the ellipsis of a higher phase CLP, (29b) is the ellipsis of a lower phase KP or the ellipsis of the complement of the head of a higher phase CLP and (29c) is the ellipsis of the complement of the head of a lower phase KP. We can also get another conclusion: There can exist more than one phase in Chinese complex NP and phases are hierarchical. Beyond the lower phase, one or more than one higher phase can exist.

Next we can use this modified theory to analyze another complex NP:

(32) Wo xihuan Zhangsan de na jian hongse de maoyi,

I like Zhangsan $\mathrm{K}$ that $\mathrm{CL}$ red $\mathrm{K}$ sweater,

a. bu xihuan [Lisi de na jian hongse de maoyi].

b.bu xihuan [Lisi de na jian hongse de maøyi].

c. bu xihuan [Lisi de na jian hongse de maoyi].

not like Lisi K that CL red K sweater.

I like that red sweater of Zhangsan's, but I don't like that red sweater of Lisi's.

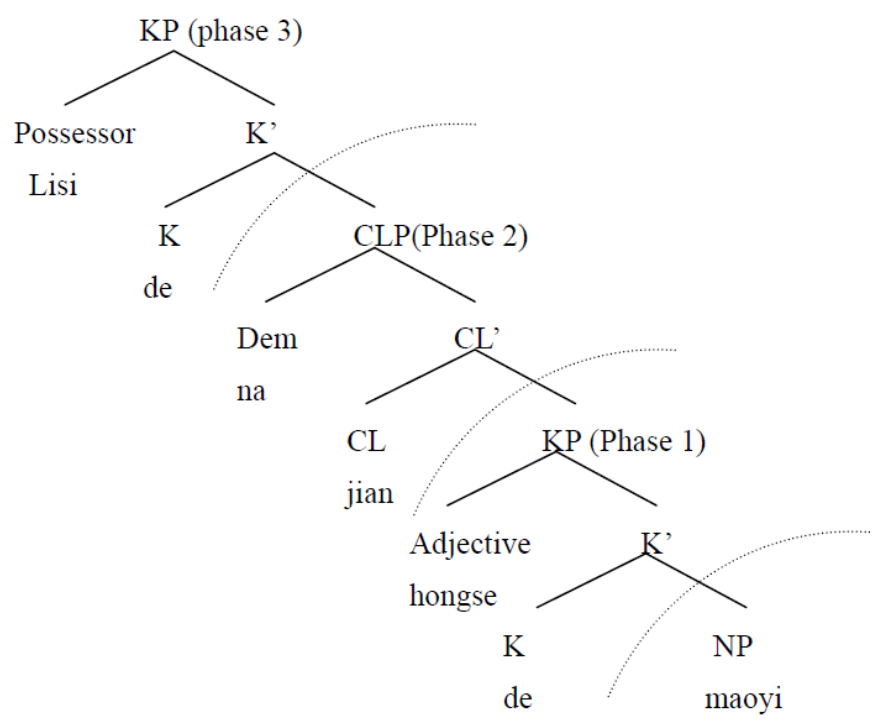


The grammaticality of (32a) (32b) and (32c) can be justified in the following way:

Form the highest layer of phases, (32a) is the ellipsis of the complement of the head of phase 1; (32b) is the ellipsis of phase 1 or the ellipsis of the complement of the head of phase $2 ;(32 \mathrm{c})$ is the ellipsis of phase 2 or the ellipsis of the complement of the head of phase 3 .

To sum up, we can say that Bošković's highest-phrase-as-a-phase approach and ellipsis-constrained-by-phase approach work well for the analysis of Chinese simple NPs, however there is a flaw to analyze the Chinese complex NPs. In order to make these two theories to have greater explanation force, we need to modifier it a little and this modified theory can also works well for the analysis of Chinese simple NPs. Because in Chinese simple NPs, there is only one functional phrase over NP, according to our modified hypotheses in (31), this functional phrase is a phase. Ellipsis of FunctionalP or Ellipsis of the complement of this FunctionalP that is NP is possible. After careful discussion, we find that Bošković's hypotheses can only explain Chinese simple NPs perfectly but they cannot explain Chinese complex NP well, however our modified hypotheses can account for not only Chinese complex NPs but also Chinese simple NPs. In this way, we can use the modified theory to account for the phasehood of Chinese NPs and the ellipsis of Chinese NPs:

(31) a. Only phases and complements of phase heads can undergo ellipsis.

b. Each functional projection over NP in Chinese is a phase.

\section{SUMMARY}

In this paper, we explore the syntactic structure of Chinese NPs and the ellipsis of Chinese NPs within two broad Chinese NPs: Chinese simple NPs and Chinese Complex NPs. We establish a unified structure for the four basic Chinese simple NPs, that is,

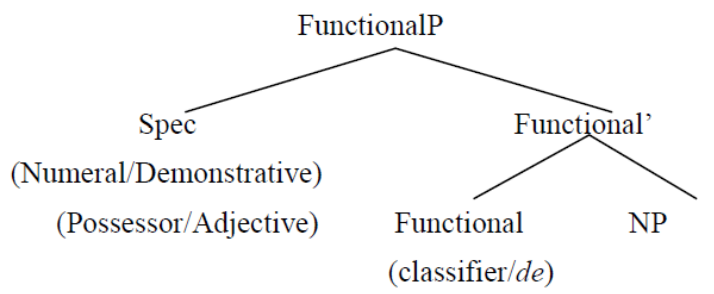

We also uncover that more than one functional phrase in Chinese complex NPs exist and this provides a hindrance for the explanation of Bošković's phase theory and ellipsis theory.

Bošković proposes that phase is the highest projection in the extended projection of a major category, and he also proposes that ellipsis is a phase-based operation. In this paper I demonstrate how these two proposals can account for Chinese simple and complex NPs and the ellipsis phenomena within Chinese NPs. By analysis of Chinese NPs, I find that Bošković's two theories can work well in the explanation of Chinese simple NPs. However when we come to Chinese complex NPs, we discover that these two theories become a little helpless. So according to the feature of Chinese NPs, we alter the two theories especially the ellipsis theory a little:

a. Only phases and complements of phase heads can undergo ellipsis.

b. Each functional projection over NP in Chinese is a phase.

\section{REFERENCES}

[1] Abney, S.P. (1987). The English noun phrase in its sentential aspect. Doctoral dissertation. MIT.

[2] Au-Yeung, B. W.H. (2001). Numerals in the classifier phrase of Chinese. Paper presented at the First International Conference on Formal Linguistics (China), Hunan China, 9-11 June 2001.

[3] Bošković, Ž. (2008). What will you have, DP or NP? In E. Elfner \& M. Walkow (Eds.), Proceeding of the North East Linguistics Society 37. Amherst: UMASS, 101-114.

[4] Bošković, Ž. (2009a). More on the NP-DP analysis of article-less languages. Studia Linguistica, 63, 187-203.

[5] Bošković, Ž. (2012). On NPs and clauses. In G. Grewenforf \& T. E. Zimmermann (Eds.), Discourse and grammar: from sentence types to lexical categories. Berlin: De Gruyiter Mouton, 179-246.

[6] Bošković, Ž. (2014). Now I'm a phase, now I'm not a phase: On the variability of phases With extraction and ellipsis. Linguistic Inquiry, 45, 27-89. http://www.mitpressjournals.org/doi/abs/10.1162/LING_a_00148\#.U09BOBafeL0 (accessed 01/02/2014).

[7] Bošković, $\quad$ Ž. $\quad \& \quad$ Serkan, $\quad$ Ş. $\quad$ (in $\quad$ press). $\quad$ The $\quad$ Turkish http://web2.uconn.edu/boskovic/papers/Boskovic\&Sener.v4.-1FinalEdited.pdf. (accessed 11/12/2013).

[8] Chao, Y. R. (1968). A grammar of spoken Chinese. Berkeley and Los Angeles, California: University of California Press.

[9] Cheng, L. L-S. \& Sybesma, R. (1999). Bare and Not-so-bare nouns and the structure of NP. Linguistic Inquiry, 30, $509-542$.

[10] Chierchia, Gennaro. (1998). Reference to kinds across languages. Natural Language Semantics, 6, 339-405.

[11] Cinque, G. (1995). Italian syntax and universal grammar. Cambridge: Cambridge University Press.

[12] Crisma P. (1993). On adjective placement in Romance and in Germanic event nominals. Rivista di Grammatica Generativa, 18, $61-100$.

[13] Fukui, N. (1995). Theory of projection in syntax. California: CSLI Publications. 
[14] Gao, Q. (1994). Chinese NP structure. Linguistics, 22, 475-510.

[15] Ionin, T. \& Ora, M. (2004a). A healthy twelve patients. Paper presented at GURT 2004.

[16] Ionin, T. \& Ora, M. (2004b). The non-existence, syntax, and semantics of plurals. USC \& CNRS/Universit Paris 8/ENS Ms.

[17] Jackendoff, R. (1971). Gapping and related rules. Linguistic Inquiry, 2, 21-35.

[18] Laenzlinger, C. (2000). More on adverb syntax. In A. Alexiadou \& P. Svenonius (Eds.) Linguistics in Potsdam (vol. 6). Potsdam: University of Potsdam, 103-132.

[19] Partee, B. H. (1987). Noun phrase interpretation and type shifting principles. In J. A. G. Groenendijk, D.H. J. Jongh \& M.J. B. Stokhof (Eds.), Studies in discourse representation theories and the theory of generalized quantifiers. Dordrecht: Foris, 115-143.

[20] Partee, B. H. (2005). Reflections of a formal semanticist as of Feb 2005. http://people.umass.edu/partee/docs/BHP_Essay_Feb05.pdf. (accessed 08/12/2013).

[21] Pysz, A. (2006). The structural location of adnominal adjectives: Prospects for Old English. SKASE Journal of Theoretical Linguistics, 3, 59-85.

[22] Saito, M., Lin, J. \& Murasugi K. (2008). N'-ellipsis and the structure of noun phrases in Chinese and Japanese. Journal of East Asian Linguistics, 17, 247-271.

[23] Szabolcsi, A. (1994). The noun phrase. In F. Kiefer \& K. E. Kiss (Eds.), Syntax and semantics: The syntactic structure of Hungarian (vol. 27). San Diego: Academic Press, 179-274.

[24] Takahashi, M. (2011). Some consequences of case-marking in Japanese. Doctoral dissertation. University of Connecticut.

[25] Tang, J. C-C. (1990). Chinese phrase structure and the extended X' theory. Doctoral dissertation. Cornell University.

[26] Xue, P. \& McFetridge, P. (1998). Verb complementation, null pronominals and binding. In E. Curtis, J. Lyle \& G. Webster (Eds.), Proceedings of the Sixteenth West Coast Conference on Formal Linguistics. Stanford, CA: Center for the Study of Language and Information, 479-493.

Cunying Fan was born in Weifang, China. She received her M.A. in linguistics from Qufu Normal University, China in 2006.

She is currently studying for her doctoral degree in linguistics at Department of English Language \& Literature, Gyeongsang National University, South Korea. Her research interests include Syntax and Discourse Analysis. 